\title{
The Effects of an Unloading Knee Brace and Insole with Subtalar Strapping for Medial Osteoarthritis of the Knee
}

\author{
Masataka Deie $^{1}$, Hayatoshi Shibuya ${ }^{2}$, Atsushi Okuhara ${ }^{2}$, Minoru Toriyama ${ }^{1}$, Nobuyuki Kumahashi ${ }^{3}$, \\ Yuji Uchio $^{3}$, Yoshiaki Sasashige ${ }^{4}$, Mitsuo Ochi ${ }^{2}$ \\ ${ }^{1}$ Department of Physical Therapy and Occupational Therapy Sciences, Graduate School of Health Sciences, Hiroshima University, \\ Hiroshima, Japan; ${ }^{2}$ Department of Orthopaedic Surgery, Graduate School of Biomedical Sciences, Hiroshima University, Hiroshima, \\ Japan; ${ }^{3}$ Departement of Orthopaedics, Shimane University, Izumo, Japan; ${ }^{4}$ Chugoku Rosai Hospital, Kure, Japan. \\ Email: snm3@hiroshima-u.ac.jp
}

Received October $15^{\text {th }}, 2013$; revised November $5^{\text {th }}$, 2013; accepted November $30^{\text {th }}, 2013$

Copyright (c) 2013 Masataka Deie et al. This is an open access article distributed under the Creative Commons Attribution License, which permits unrestricted use, distribution, and reproduction in any medium, provided the original work is properly cited. In accordance of the Creative Commons Attribution License all Copyrights (C) 2013 are reserved for SCIRP and the owner of the intellectual property Masataka Deie et al. All Copyright (C) 2013 are guarded by law and by SCIRP as a guardian.

\begin{abstract}
Objectives: We evaluated whether a valgus knee brace and an insole with subtalar strapping could reduce pain and improve functional scores over 12 months in patients with medial knee osteoarthritis (OA). Methods: OA was confirmed by radiography in all patients, who were divided into three groups: exercise $(n=44)$, insole $(n=55)$, and brace $(n=19)$. Clinical knee functions and gait analyses were evaluated. Results: After 12 months, the clinical results for all groups had improved compared to pretreatment findings. Although the knee varus moment decreased and the gait speed increased when the insole was worn initially, the effects of the insole decreased at 6 and 12 months. In contrast, the knee varus moment decreased and the gait speed increased when the brace was initially fitted. The effects of the brace were maintained during the 12 months. Conclusions: The insole was effective for patients with Kellgren-Lawrence Grades II and III, and the knee brace was effective for patients with Grades III and IV conditions. Furthermore, the knee brace was more effective when worn for more than 6 months, while the effects of the insoles were not continuous.
\end{abstract}

Keywords: Medial Osteoarthritis; Insole; Functional Brace; Knee Varus Moment; Gait Analysis

\section{Introduction}

Patients with knee osteoarthritis (OA) are encountered increasingly in our aging population. Patients with medial knee OA present varus deformities and increased mechanical stresses in the medial compartment $[1,2]$, which together increase pain and functional disabilities [3]. Varus knee deformities cause patients to shift the lower limb axis to the medial compartment and walk with greater peak external knee adduction moment compared to individuals without knee OA [4]. The treatment for varus knee deformities can initially involve various conservative approaches such as muscle-strengthening exercises, medicines, and orthoses. A systematic review regarding the benefits of exercise for knee OA [5] revealed no definitive proof that such exercises are effective in preventing the progression of knee OA. However, the current guidelines for OA [6,7] indicate that musclestrengthening exercises and medicines effectively reduce knee joint pain and aid the recovery of knee joint function.

The effects of orthoses on knee OA are also controversial. Unloading knee braces, which are recommended for the non-pharmacological management of knee OA by EULAR [8], may relieve short- and mid-term pain more effectively than neoprene knee sleeves. They can also provide short-term improvements infunctional disabilities and quality of life for knee OA patients [9]. Previous studies demonstrated that braces treat knee OA by extending the condylar separation of the medial compartment [10], thus reducingthe external knee adduction moment during gait [11-13] and the medial compartment load at the knee $[13,14]$. In the clinical setting, many patients treated with knee bracing complained that the braces were heavy and complicated to fit, and that longterm fittings caused skin problems. Recently, lighter and more comfortable knee braces were developed; however, 
the effects of these new functional knee braces are not well known.

The effects of insoles, which are one of the main orthoses used to treat knee OA, are also controversial. Yasuda and Sasaki first described the function and effects of the lateral-wedge insole in 1987 [15], and Toda and Tsukimura [16] subsequently reported that lateral-wedge insoles with subtalar strapping were more effective than conventional insoles in alleviating the symptoms of knee OA. Lateral-wedge insoles show considerable potential in the treatment of symptomatic knee OA because they are safe and generate fewer costs than knee bracing. Furthermore, foot orthoses are easy to apply, and good patient adherence to this type of intervention has been reported $[17,18]$. However, recent studies have suggested that lateral-wedge insoles are not particularly effective for treating knee OA $[19,20]$.

This 12-month prospective study therefore sought to clarify the effects of muscle-strengthening exercises, the unloading knee brace, and insole with subtalar strapping on medial OA of the knee by assessing clinical function and gait. We hypothesized that these orthoses could provide OA patients with relief from knee pain and improve knee function compared to muscle-strengthening exercises only.

\section{Methods}

This study was approved by the Epidemiologic Study Ethics Review Board of Hiroshima University and conformed to the Declaration of Helsinki.

\section{Patients}

A total of 151 patients who were diagnosed with medial compartment OA of the knee between June 2008 and December 2009, and who agreed to this study, were enrolled. These patients presented with knee OA symptoms including pain and functional disabilities. Medial compartment OA of the knee was confirmed by radiography. Patients were excluded if they had any of the following: history of lower limb injuries within the last 2 years, a neuromuscular disorder, history of stroke or cardiovascular disease, or any other gait abnormalities. Severity of knee OA was assessed by radiography and rated using the Kellgren-Lawrence radiographic scoring system (K-L scores) [21]. Patients assigned a K-L score of grade IIor higher were selected and informed as to the aim of this prospective study. Finally, 118 patients with medial compartment OA of the knee were enrolled in the study. The three different treatment methods were described to the patients, who then selected their treatment and the side to be evaluated. Accordingly, the patients were divided into three groups: the exercise, insole, and brace groups. The exercise group comprised 44 patients who were asked to perform three sets of 10 quadriceps muscle exercises every day. The insole group comprised 55 patients who were advised to wear the lateral-wedge type of insole with subtalar strapping (Figure 1) for more than 5 hours every day. This type of insole was designed specifically for patients with knee OA. The brace group comprised 19 patients who were advised to wear a functional knee brace for more than 5 hours every day. The functional knee brace used in this study was the Unloader ${ }^{\circledR}$ One (Össur, Reykjavik, Iceland; Figure 2), whichwas designed specifically for patients with knee OA and combines a streamlined, flexible upright with a medial hinge to create two leverage points when opposing forces from the Dynamic Dual Force Straps, which cross over the knee, disperse a counter force across two lateral-aspect points of the knee. The brace is an off-the-shelf patientadjustable device composed mainly of nylon, measuring approximately $367 \mathrm{~mm}$ in length, and weighing approximately $430 \mathrm{~g}$. The brace is also available in three sizes (XS, S, M).

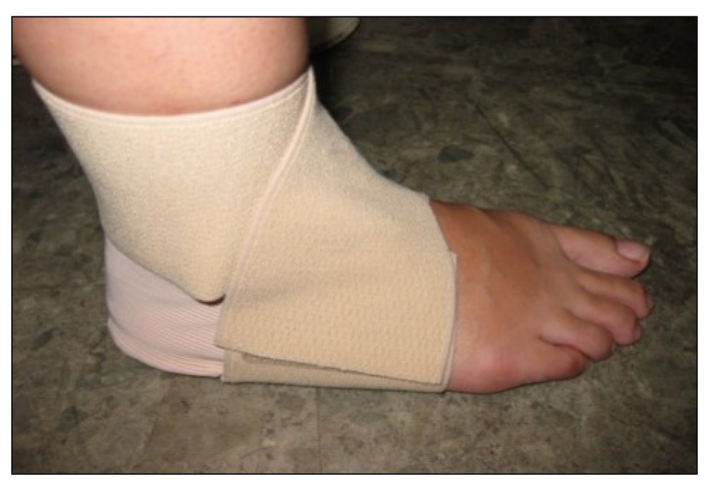

Figure 1. Lateral view of an insole used in this study, showing the conventional insole with subtalar strapping.

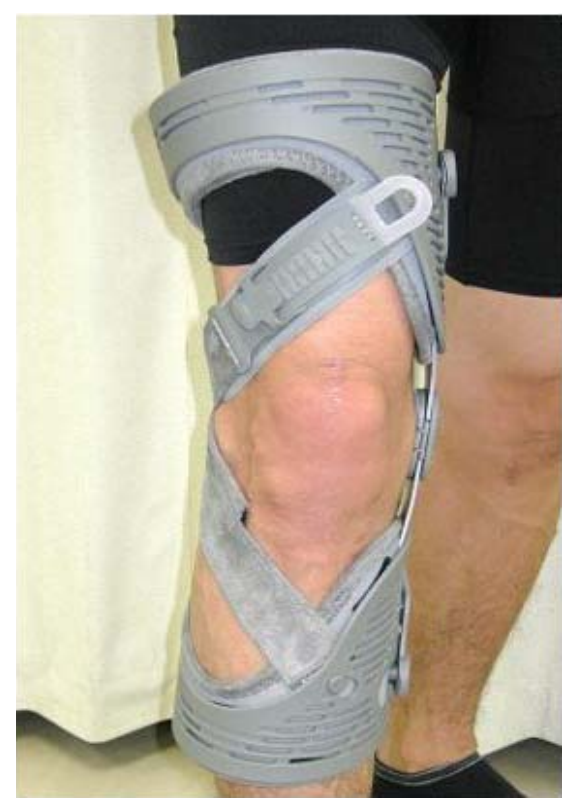

Figure 2. Front view of the functional knee brace, Unloader ${ }^{\circledR}$ One (Össur, Reykjavik, Iceland). 


\section{Evaluations}

\section{Physical evaluations}

Clinical findings such as range of motion (ROM), quadriceps muscle power, and $\mathrm{x}$-rays with K-L scores were evaluated as objective findings, especially for the medial femoro-tibial joints. Original questionnaire results, based on the SF-8 Health Survey (SF-8), Western Ontario and McMaster Universities Arthritis Index (WOMAC), and EuroQol 5 demension (EQ-5D) questionnaires, were also evaluated before treatment and at 3, 6, and 12 months after treatment. These indices are used generally to assess patients with osteoarthritis, and can be used to monitor the course of the disease. SF- 8 reflects the state of the patients over one month, WOMAC reflects a 48-hour period, and EQ-5D reflects the patient state over 24 hours.

\section{Gait evaluations}

In total, 31 patients agreed to undergo gait analysis evaluation, including 9 patients in the exercise group, 12 in the insole group, and 10 in the brace group. The patients were examined be fore treatment, then at 6 months and 12 months after treatment. The patients' gaits were analyzed using a three-dimensional motion analysis system (VICON612, Vicon Motion Systems Ltd, Oxford, UK) with seven infrared cameras (Vicon Motion Systems Ltd, Oxford, UK) operating at $120 \mathrm{~Hz}$. Kinetic data were captured from the ground reaction forces at $500 \mathrm{~Hz}$ with four force platforms (AMTI, Watertown, MA) embedded in the rehabilitation room floor and synchronized to the kinematic data. Passive reflective markers were placed bilaterally on the anterior and posterior iliac spines, lateral aspects of the thighs, lateral femoral epicondyles, lateral aspects of the shanks, lateral malleoli, calcanea, and second metatarsal heads according to a commercially available kinematic model (Plug-in-Gait, Vicon ${ }^{\circledR}$ Peak, Oxford, UK). Patients were instructed to walk at a selfselected velocity along a 10 -meter walkway. Patients walked without orthoses and then with bracing or insoles on the more painful side at each evaluation time point. This was conducted at least three times for each condition.

\section{Gait data analysis}

Marker coordinate data and ground reaction data were low-pass filtered (Butterworth fourth-order filter, cut-off frequency $6 \mathrm{~Hz}$ ) with plug-in software (ButterPlug, Vaquita Software, Zaragoza, Spain). Knee and hip joint angles and moment data were calculated using a comercially available biomechanical model (Plug-in-Gait, Vi$\operatorname{con}^{\circledR}$ Peak, Oxford, UK). Joint moment data were analyzed in the frontal planes in the lower limb. The knee adduction moment impulse, the timed integral of all the knee adduction moments, was calculated for stance duration and the subdivisions of stance duration. Joint moment data were extracted for statistical analysis and nor- malized to body mass $(\mathrm{Nm} / \mathrm{kg})$. Walking speed, cadence, step length, and stride length were extracted from the biomechanical model output.

\section{Statistical analysis}

\section{Gait data}

Data fitting a normal distribution were analyzed by the paired samples $t$-test. Data that were not normally distributed were analyzed for significant differences in the joint angle and moment by the Wilcoxon signed-rank test, using statistical analysis software (SPSS v16.0, SPSS Japan, Tokyo, Japan). Results were considered to be significant when $P<0.05$.

\section{Physical data}

To determine the independent and combined effects of normally distributed variables (walking visual analogue scale (VAS), resting VAS, femoro-tibial angle (FTA), extension ROM, and flexion ROM), comparisons between the groups and over time were performed using two-way ANOVA. When significant effects or interactions were found, one-way ANOVA was followed by the Bonferroni post-hoc test to identify the levels off actor(s) with significant differences in the means of the variables. These variables are presented as the mean \pm standard error of the mean (SEM).

Group comparisons of these categorical variables and $\mathrm{K}-\mathrm{L}$ grades were performed using the Kruskal-Wallis $\mathrm{H}$ test. The Friedman Repeated Measures ANOVA on Ranks test was used to analyze changes over time within each group. When statistical significance was achieved, a SteelDwass post-hoc test was used to further specify the difference. Results were considered to be significant when $P<0.05$.

\section{Results}

\section{Patient characteristics}

There were no differences in sex, age, height, weight, or body mass index among the exercise, insole, and brace groups (Table 1).

\section{$X$-ray evaluations}

There were significantly more patients with Grade II $\mathrm{OA}$ in the exercise and insole groups than in the brace group. In contrast, there were significantly more patients

Table 1. There were no differences in sex, age, height, weight, or body mass index among the exercise, insole, and brace groups.

\begin{tabular}{cccc}
\hline & Exercise & Insole & Brace \\
\hline Patients (n) & 44 & 55 & 19 \\
Male: Female & $18: 26$ & $25: 30$ & $8: 11$ \\
Age (y) & $65 \pm 2.1$ & $67 \pm 3.4$ & $69 \pm 4.2$ \\
Height (cm) & $152.6 \pm 3.2$ & $153.1 \pm 2.6$ & $152.6 \pm 2.5$ \\
Weight (kg) & $57.6 \pm 1.7$ & $57.5 \pm 1.4$ & $60.5 \pm 4.4$ \\
BMI (Body Mass Index) & $24.9 \pm 1.5$ & $24.6 \pm 1.7$ & $26.2 \pm 2.3$ \\
\hline
\end{tabular}


with Grades III and IV OA in the brace group compared to the exercise and insole groups (Figure 3). At the final evaluation there was only one progressive case, in the exercise group.

\section{Clinical analysis}

\section{Subjective findings}

There were no statistical changes in the results of the WOMAC, SF-8 and EQ-5D questionnaires completed before treatment, and at 3 months, 6 months, and 12 months after treatment. The walking VAS scores improved in all three groups. Although the walking VAS scores of the brace group were significantly higher than those of the exercise group before treatment commenced $(P<0.05)$, the significant difference among these three groups disappeared after treatment (Figure 4).

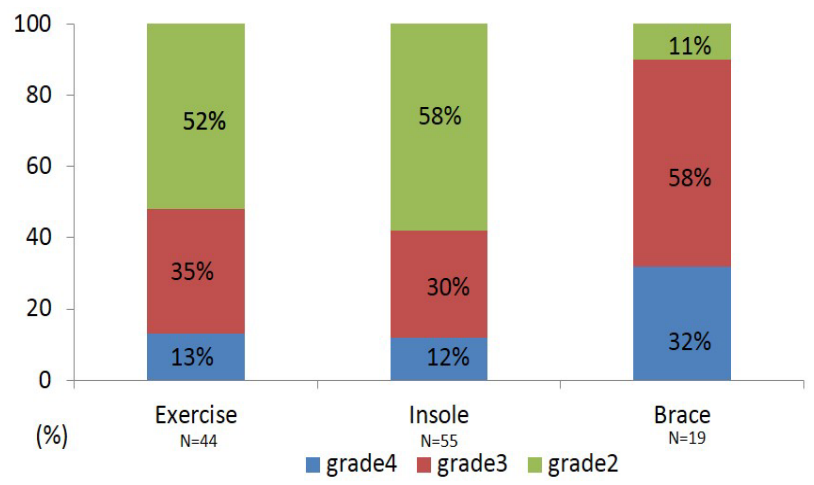

Figure 3. Kellgren-Lawrence classification for patients in each of the three groups. There were significantly more patients with Grade II OA in the exercise and insole groups than in the brace group. In contrast, there were significantly more patients with Grades III and IV OA in the brace group compared to the exercise and insole groups.

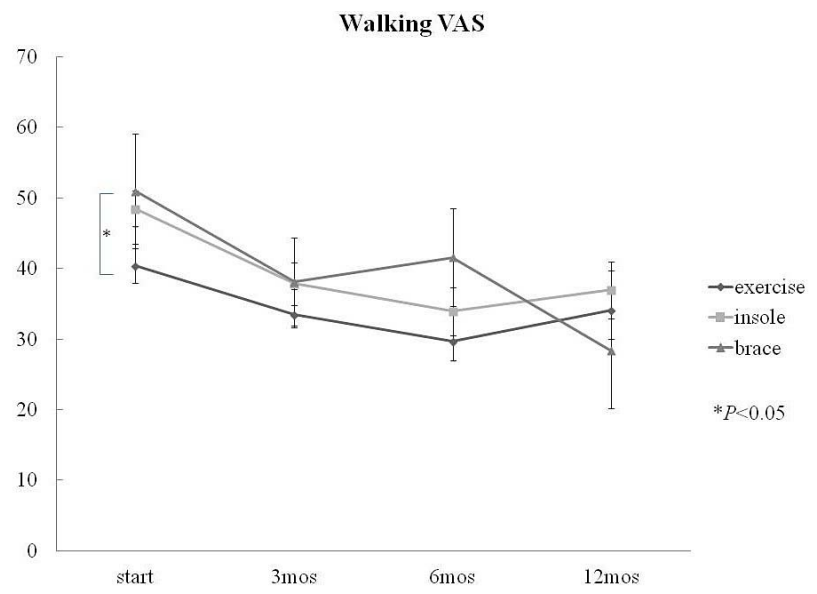

Figure 4. The walking VAS score. The graph shows a decrease in VAS scores during walking in each group. Although the walking VAS scores of the brace group were significantly higher than those of the exercise group before treatment commenced $(P<0.05)$, there was no significant difference in VAS scores among the three groups after treatment.

\section{Objective findings}

Gait length and ability to walk up and down stairs improved in all three groups, with the greatest improvement seen in the brace group. Patients in the brace groups showed improved knee extension angles, but significantly worse extension disturbance, than those in the exercise and insole groups at each of the follow-up times $(P<0.05)$ (Figure 5). Although the FTA of the brace group was larger than that of the exercise group at all times, the FTA of the three groups did not significantly change at any time.

\section{Drop out cases}

A total of 24 patients dropped out of this study, including 10 cases (22.7\%) in the exercise group, 13 cases (23.6\%) in the insole group, and one case $(5.3 \%)$ in the brace group.

\section{Gait analysis}

The varus moment did not significantly improve in any of the three groups over the 12 months. However, the changes in the ratios of the varus moment before and after the orthoses were worn show the difference between the brace and insole. Initially, the knee varus moment decreased by $9.5 \%$ when the insole was worn, but at 6 months and 12 months the knee varus moment only decreased by $0.5 \%$ and $0.7 \%$, respectively. In contrast, the varus moment of the knee decreased $5.5 \%$ when the brace was initially fitted. This decreased ratio was maintained during the 12 months (Figure 6).

The walking speed did not significantly improve in any of the three groups either. However, the changes in the ratios of the gait speed before and after the orthoses were worn show the difference between the brace and insole. At the start, the gait speed increased $12.3 \%$ when wearing the insole, but at 6 months and 12 months, the gait speed did not increase when the insole was worn. On

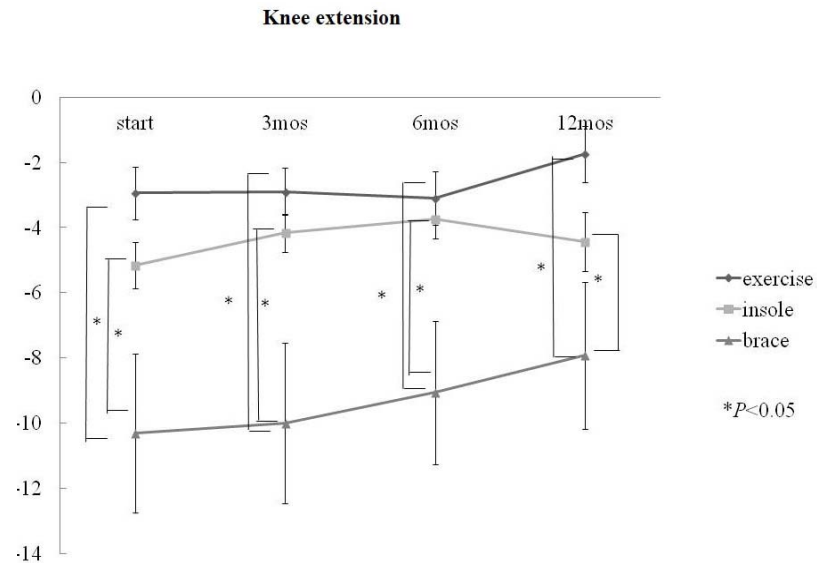

Figure 5. Knee extension angle. The graph shows that while the patients in the brace group had significantly worse extension disturbance than those in the exercise and insole groups at each of the follow-up times, the extension angle in the brace group was gradually improved. 


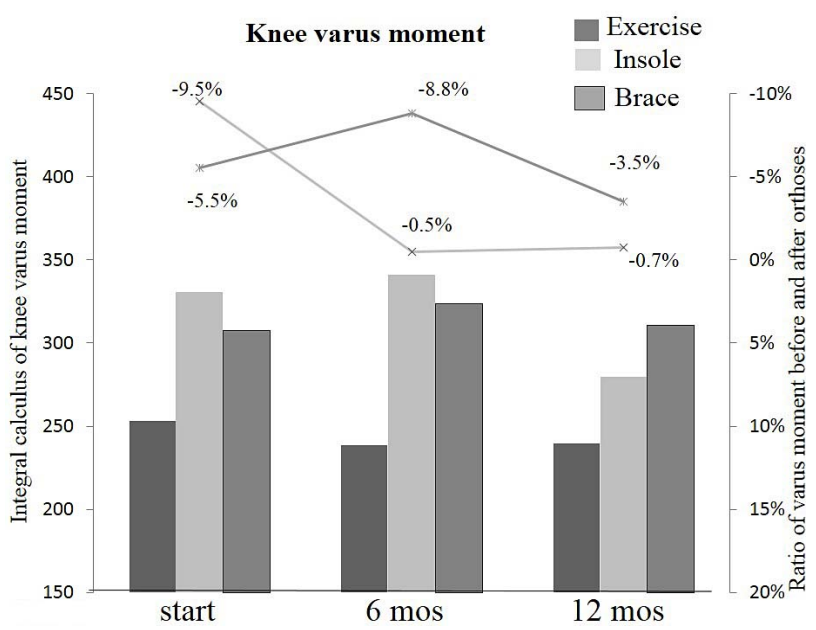

Figure 6. Knee varus moment for the three groups over 12 months. The bar graph comparing the knee varus moment during free gait shows no significant improvements in any of the three groups over the $\mathbf{1 2}$ months. The line traces at the top represents the changes in ratios before and after the orthoses were worn. The varus moment decreased by $9.5 \%$ when the insole was worn, but at 6 months and 12 months the knee varus moment had only decreased further by $\mathbf{0 . 5 \%}$ and $0.7 \%$, respectively. The varus moment decreased by $5.5 \%$ when the brace was initially fitted, and this decreased ratio was maintained during the 12 months.

the other hand, the gait speed initially increased by $16.4 \%$ when the brace was fitted, and the gait speed at 6 months and 12 months was much higher than seen at the start of the study (Figure 7).

\section{Discussion}

There have been several recent reports about knee orthoses. However, to our knowledge, this is the first prospective study describing the effects of a functional brace, a type of insole, and muscle-strengthening exercises on the osteoarthritic knee joint. The patients in the exercise group had significantly lower grade OA than those in the brace group. Patients in the exercise group demonstrated improved gait ability and decreased resting pain after performing quadriceps exercises for one year. The insole group included many patients with low and middle-grade $\mathrm{OA}$, and following treatment the patients demonstrated an improved ROM and ability to climb up and down stairs. The patients in the brace group mainly had significantly severe OA, yet treatment still improved their walking VAS, ability to climb up and down stairs, and ROM. These results demonstrate that conservative treatments for medial knee OA, such as muscle-strengthening exercises, the insole, and knee brace, effectively improved gait ability and decreased resting and walking pain when applied to patients according to their OA grades. This study shows that the symptoms in patients with severe OA also improved after treatment with the valgus brace.

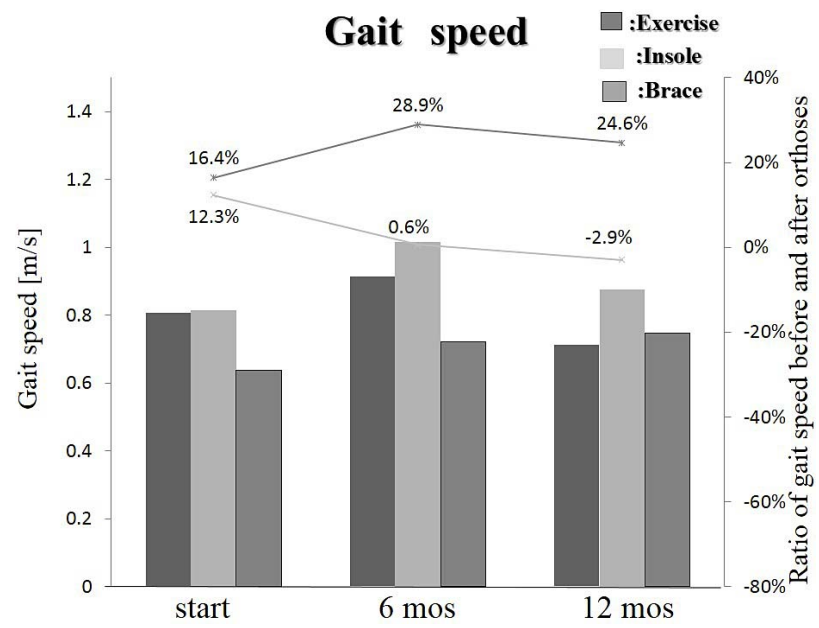

Figure 7. Gait speed for the three groups over 12 months. The bar graph comparing the gait speed during free gait shows no significant improvements in any of the three groups over the 12 months. The line traces at the top represent the changes in ratios before and after the orthoses were worn. The gait speed increased $12.3 \%$ when wearing the insole, but at 6 months and 12 months, no further increase was achieved. The gait speed initially increased by $16.4 \%$ when the brace was fitted, and continued to increase at 6 months and 12 months.

The evidence presented in previous studies regarding whether foot orthoses or knee braces improved pain and function in patients with medial knee OA has been controversial $[15,22,23]$. Lateral-wedge insoles that did not cover the subtalar joint were used in many of these previous studies. Toda et al. $[16,17]$ reported that the new type of insole was more effective than the old ones. They demonstrated that the new insole changed the FTA in medial knee OA patients and used weight-bearing radiographs to show how the new insole corrected the FTA by 3 degrees. Furthermore, Kuroyanagi et al. [23] reported that the new type of insole was more effective at reducing pain than the conventional insole during gait, as measured by the gait analysis method. They also reported that no significant difference was detected between the new type of insole and the conventional insole when worn by patients with severe OA (K-L Grade 4). The results from these previous reports and the current study indicate that this type of insole would be effective for patients with Grade II or III OA. In contrast, the brace group consisted of patients with Grade III or IV OA, the walking VAS, extension lag, ability to climb up and down stairs, and ROM improved with treatment. These results indicated that the functional knee brace effectively relieved pain and improved knee motion function and gait ability in patients with severe OA.

The dropout rate in this study was not satisfactory, with over $20 \%$ of patients in the insole and exercise groups not continuing. This indicated that motivation to 
continue exercising must be maintained and patient compliance with wearing the new type of insole must be improved. In contrast, there was only one dropout case in the brace group, which could reflect several improvements in this functional knee brace that reduced the weight by approximately $50 \%$ compared to a conventional functional knee brace, and made it easier to wear.

Gait analysis was evaluated for each patient before treatment and at 3 months, 6 months and 12 months after treatment. The results revealed that the effects of the insole gradually decreased; however, the effects of the brace increased. These results showed that patients became used to the fit of the brace and learned to walk wearing the brace over time, whereas the effects of the insole were not maintained. We recommend that the insole should be exchanged every three months, but the functional brace can be worn for more than one year.

There were some limitations associated with this study. Firstly, this study was prospective in nature, rather than being a randomized control study. Secondly, patients each chose their own treatment. The exercise group was composed of K-L Grade II patients and the brace group comprised K-L Grade III or IV patients. Finally, the exercise group is not a true control group, and it remains difficult to compare non-treatment patients in such studies to those receiving treatment. However, in this current study we could conclude that the orthotic treatments were more effective at treating medial compartment OA of the knee when the treatment procedures were selected according to the OA grade.

\section{Acknowledgements}

This paper was supported by the following grants: JOASubsidized Science Project Research 2007-3 and Comprehensive Research on Aging and Health from the Health and Labor Sciences Research Grants.

\section{REFERENCES}

[1] L. Weidenhielm, O. K. Svensson, L. A. Brostrom and E. Mattsson, “Adduction Moment of the Knee Compared to Radiological and Clinical Parameters in Moderate Medical Osteoarthrosis of the Knee," Annales Chirurgiae et Gynaecologiae, Vol. 83, No. 3, 1994, pp. 236-242.

[2] T. P. Andriacchi, A. Mundermann, R. L. Smith, E. J. Alexander, C. O. Dyrby and S. Koo, "A Framework for the in vivo Pathomechanics of Osteoarthritis at the Knee," Annals of Biomedical Engineering, Vol. 32, No. 3, 2004, pp. 447-457. http://dx.doi.org/10.1023/B:ABME.0000017541.82498.3 $\underline{7}$

[3] L. Sharma, J. Song, D. T. Felson, S. Cahue, E. Shamiyeh and D. D. Dunlop, "The Role of Knee Alignment in Disease Progression and Functional Decline in Knee Osteoarthritis,” JAMA, Vol. 286, No. 2, 2001, pp. 188-195. http://dx.doi.org/10.1001/jama.286.2.188

[4] A. J. Baliunas, D. E. Hurwitz, A. B. Ryals, A. Karrar, J. P. Case, J. A. Block and T. P. Andriacchi, "Increased Knee Joint Loads during Walking Are Present in Subjects with Knee Osteoarthritis,” Osteoarthritis Cartilage, Vol. 10, No. 7, 2002, pp. 573-579.

http://dx.doi.org/10.1053/joca.2002.0797

[5] M. Fransen and S. McConnell, "Exercise for Osteoarthritis of the Knee," Cochrane Database of Systematic Reviews, Vol. 8, No. 4, 2008, Article ID: CD004376. http://dx.doi.org/10.1002/14651858.CD004376.pub2

[6] American Academy of Orthopaedic Surgeons, "Treatment of Osteoarthritis of the Knee (Non-Arthroplasty),” Rosemont (IL) American Academy of Orthopaedic Surgeons (AAOS), 2008, pp. 30-41.

[7] W. Zhang, R. W. Moskowitz, G. Nuki, S. Abramson, R. D. Altman, N. Arden, S. Bierma-Zeinstra, K. D. Brandt, P. Croft, M. Doherty, M. Dougados, M. Hochberg, D. J. Hunter, K. Kwoh, L. S. Lohmander and P. Tugwell, "OARSI Recommendations for the Management of Hip and Knee Osteoarthritis, Part II: OARSI Evidence-Based, Expert Consensus Guidelines,” Osteoarthritis Cartilage, Vol. 16, No. 2, 2008, pp. 137-162. http://dx.doi.org/10.1016/j.joca.2007.12.013

[8] K. M. Jordan, N. K. Arden, M. Doherty, B. Bannwarth, J. W. Bijlsma, P. Dieppe, K. Gunther, H. Hauselmann, G. Herrero-Beaumont, P. Kaklamanis, S. Lohmander, B. Leeb, M. Lequesne, B. Mazieres, E. Martin-Mola, K. Pavelka, A. Pendleton, L. Punzi, U. Serni, B. Swoboda, G. Verbruggen, I. Zimmerman-Gorska and M. Dougados, "EULAR Recommendations 2003: An Evidence Based Approach to the Management of Knee Osteoarthritis: Report of a Task Force of the Standing Committee for International Clinical Studies Including Therapeutic Trials (ESCISIT)," Annals of the Rheumatic Diseases, Vol. 62, No. 12, 2003, pp. 1145-1155. http://dx.doi.org/10.1136/ard.2003.011742

[9] J. Beaudreuil, S. Bendaya, M. Faucher, E. Coudeyre, P. Ribinik, M. Revel and F. Rannou, "Clinical Practice Guidelines for Rest Orthosis, Knee Sleeves, and Unloading Knee Braces in Knee Osteoarthritis,” Joint Bone Spine, Vol. 76, No. 6, 2009, pp. 629-636. http://dx.doi.org/10.1016/j.jbspin.2009.02.002

[10] R. D. Komistek, D. A. Dennis, E. J. Northcut, A. Wood, A. W. Parker and S. M. Traina, "An in Vivo Analysis of the Effectiveness of the Osteoarthritic Knee Brace during Heel-Strike of Gait,” The Journal of Arthroplasty, Vol. 14, No. 6, 1999, pp. 738-742. http://dx.doi.org/10.1016/S0883-5403(99)90230-9

[11] T. N. Lindenfeld, T. E. Hewett and T. P. Andriacchi, "Joint Loading with Valgus Bracing in Patients with Varus Gonarthrosis,” Clinical Orthopaedics and Related Research, Vol. 344, 1997, pp. 290-297. http://dx.doi.org/10.1097/00003086-199711000-00029

[12] R. D. Gaasbeek, B. E. Groen, B. Hampsink, R. J. van Heerwaarden and J. Duysens, "Valgus Bracing in Patients with Medial Compartment Osteoarthritis of the Knee. A Gait Analysis Study of a New Brace,” Gait Posture, Vol. 26, No. 1, 2007, pp. 3-10. 
http://dx.doi.org/10.1016/j.gaitpost.2006.07.007

[13] F. E. Pollo, J. C. Otis, S. I. Backus, R. F. Warren and T. L. Wickiewicz, "Reduction of Medial Compartment Loads with Valgus Bracing of the Osteoarthritic Knee," The American Journal of Sports Medicine, Vol. 30, No. 3, 2002, pp. 414-421.

[14] D. K. Ramsey, K. Briem, M. J. Axe and L. SnyderMackler, "A Mechanical Theory for the Effectiveness of Bracing for Medial Compartment Osteoarthritis of the Knee,” The Journal of Bone \& Joint Surgery, Vol. 89, No. 11, 2007, pp. 2398-2407. http://dx.doi.org/10.2106/JBJS.F.01136

[15] K. Yasuda and T. Sasaki, "The Mechanics of Treatment of the Osteoarthritic Knee with a Wedged Insole," Clinical Orthopaedics and Related Research, No. 215, 1987, pp. 162-172.

[16] Y. Toda and N. Tsukimura, "A 2-Year Follow-Up of a Study to Compare the Efficacy of Lateral Wedged Insoles with Subtalar Strapping and In-Shoe Lateral Wedged Insoles in Patients with Varus Deformity Osteoarthritis of the Knee," Osteoarthritis Cartilage, Vol. 14, 2006, pp. 231-237. http://dx.doi.org/10.1016/j.joca.2005.09.006

[17] Y. Toda, N. Segal, A. Kato, S. Yamamoto and M. Irie, "Correlation between Body Composition and Efficacy of Lateral Wedged Insoles for Medial Compartment Osteoarthritis of the Knee," The Journal of Rheumatology, Vol. 29, No. 3, 2002, pp. 541-545.

[18] K. Baker, J. Goggins, H. Xie, K. Szumowski, M. LaValley, D. J. Hunter and D. T. Felson, "A Randomized Crossover Trial of a Wedged Insole for Treatment of Knee Osteoarthritis,” Arthritis \& Rheumatism, Vol. 56, No. 4, 2007, pp. 1198-1203. http://dx.doi.org/10.1002/art.22516

[19] T. M. van Raaij, M. Reijman, R. W. Brouwer, S. M. Bierma-Zeinstra and J. A. Verhaar, "Medial Knee Osteoarthritis Treated by Insole or Braces: A Randomized Trial," Clinical Orthopaedics and Related Research, Vol. 468, No. 7, 2010, pp. 1926-1932. http://dx.doi.org/10.1007/s11999-010-1274-z

[20] K. L. Bennell, K. A. Bowles, C. Payne, F. Cicuttini, E. Williamson, A. Forbes, F. Hanna, M. Davies-Tuck, A. Harris and R. S. Hinman, "Lateral Wedge Insoles for Medial Knee Osteoarthritis: 12 Month Randomized Controlled Trial,” BMJ, Vol. 342, 2011, Article ID: d2912. http://dx.doi.org/10.1136/bmj.d2912

[21] J. H. Kellgren and J. S. Lawrence, "Radiological Assessment of Osteo-Arthrosis," Annals of the Rheumatic Diseases, Vol. 16, No. 4, 1957, pp. 494-502. http://dx.doi.org/10.1136/ard.16.4.494

[22] E. R. Draper, J. M. Cable, J. Sanchez-Ballester, N. Hunt, J. R. Robinson and R. K. Strachan, "Improvement in Function after Valgus Bracing of the Knee. An Analysis of Gait Symmetry,” The Journal of Bone \& Joint Surgery, Vol. 82, No. 7, 2000, pp. 1001-1005. http://dx.doi.org/10.1302/0301-620X.82B7.10638

[23] Y. Kuroyanagi, T. Nagura, H. Matsumoto, T. Otani, Y. Suda, T. Nakamura and Y. Toyama, "The Lateral Wedged Insole with Subtalar Strapping Significantly Reduces Dynamic Knee Load in the Medial Compartment Gait Analysis on Patients with Medial Knee Osteoarthritis," Osteoarthritis Cartilage, Vol. 15, No. 8, 2007, pp. 932936. http://dx.doi.org/10.1016/j.joca.2007.02.004 